\title{
BMJ Open Preventing obesity in infants: the Growing healthy feasibility trial protocol
}

\author{
Elizabeth Denney-Wilson, ${ }^{1}$ Rachel Laws, ${ }^{2}$ Catherine Georgina Russell, ${ }^{1}$ \\ Kok-leong Ong, ${ }^{3}$ Sarah Taki, ${ }^{1}$ Roz Elliot, ${ }^{1}$ Leva Azadi, ${ }^{2}$ Sharyn Lymer, ${ }^{4}$ \\ Rachael Taylor, ${ }^{5}$ John Lynch, ${ }^{6}$ David Crawford, ${ }^{2}$ Kylie Ball, ${ }^{2}$ Deborah Askew, ${ }^{7}$ \\ Eloise Kate Litterbach, ${ }^{2}$ Karen J Campbell ${ }^{2}$
}

To cite: Denney-Wilson E, Laws R, Russell CG, et al. Preventing obesity in infants: the Growing healthy feasibility trial protocol. BMJ Open 2015;5:e009258. doi:10.1136/ bmjopen-2015-009258

- Prepublication history for this paper is available online. To view these files please visit the journal online (http://dx.doi.org/10.1136/ bmjopen-2015-009258).

Received 1 July 2015 Revised 30 September 2015 Accepted 16 October 2015

\section{CrossMark}

\section{${ }^{1}$ University of Technology Sydney, Sydney, New South Wales, Australia \\ ${ }^{2}$ Deakin University, Melbourne, Victoria, Australia ${ }^{3}$ Latrobe University, Melbourne, Victoria, Australia ${ }^{4}$ University of Sydney, Sydney, New South Wales, Australia \\ ${ }^{5}$ University of Otago, Dunedin, New Zealand ${ }^{6}$ University of Adelaide, Adelaide, Australia \\ ${ }^{7}$ Inala Indigenous Health, Brisbane, Australia}

Correspondence to Dr Elizabeth Denney-Wilson; Elizabeth.denney-wilson@uts. edu.au

\section{ABSTRACT}

Introduction: Early childhood is an important period for establishing behaviours that will affect weight gain and health across the life course. Early feeding choices, including breast and/or formula, timing of introduction of solids, physical activity and electronic media use among infants and young children are considered likely determinants of childhood obesity. Parents play a primary role in shaping these behaviours through parental modelling, feeding styles, and the food and physical activity environments provided. Children from low socioeconomic backgrounds have higher rates of obesity, making early intervention particularly important. However, such families are often more difficult to reach and may be less likely to participate in traditional programs that support healthy behaviours. Parents across all socio-demographic groups frequently access primary health care (PHC) services, including nurses in community health services and general medical practices, providing unparalleled opportunity for engagement to influence family behaviours. One emerging and promising area that might maximise engagement at a low cost is the provision of support for healthy parenting through electronic media such as the Internet or smart phones. The Growing healthy study explores the feasibility of delivering such support via primary health care services.

Methods: This paper describes the Growing healthy study, a non-randomised quasi experimental study examining the feasibility of an intervention delivered via a smartphone app (or website) for parents living in socioeconomically disadvantaged areas, for promoting infant feeding and parenting behaviours that promote healthy rather than excessive weight gain. Participants will be recruited via their primary health care practitioner and followed until their infant is 9 months old. Data will be collected via web-based questionnaires and the data collected inherently by the app itself.

Ethics and dissemination: This study received approval from the University of Technology Sydney Ethics committee and will be disseminated via peer-reviewed publications and conference presentations.

\section{Strengths and limitations of this study}

M-health intervention developed with expert content tailored to age of baby and feeding method.

- Extensive consultation with end users and primary health care providers informed development.

- Based on sound theoretical framework.

- RCT or cluster RCT design not possible within the study budget and timeframe but study will collect data to inform a full scale RCT in the future.

- Follow up for this study is limited to 9 months of age again due to study budget and timeframe. Ideally longer term follow up would be preferable to allow for the effect of the intervention to be assessed in later infancy and toddlerhood.

\section{INTRODUCTION}

Child obesity prevention strategies have tended to focus on older age groups, when many children are already overweight or obese. Indeed in Australia, 20\% of pre-school children are overweight or obese, suggesting that obesity prevention needs to begin much earlier. ${ }^{1}$ However while birth weights have remained fairly stable over the past few decades, there has been substantial increases in the number of pre-schoolers with obesity; suggesting the importance of the first few years of life in establishing healthy patterns of growth. ${ }^{2}$ Rapid weight gain in infancy is associated with obesity in childhood as well as being an independent risk factor for metabolic conditions and cardiovascular disease. The first year of life is therefore an important window for primary prevention of obesity.

A number of feeding behaviours are associated with obesity in infants, and may be modifiable. In particular, formula feeding, feeding beyond satiety, adding cereal to bottles, using feeding as a method of soothing, putting a baby to bed with a bottle and 
early introduction of solids are candidates for intervention. ${ }^{4}$ While maintaining or increasing the duration of breastfeeding has many benefits and remains a public health priority, many women wean their babies early, making bottle feeding practices an important target.

In high income countries like Australia, the UK and the USA, children from socioeconomically disadvantaged families have higher rates of obesity than those children experiencing less disadvantage. For example, the in the Longitudinal study of Australian children, the most disadvantaged (highest quintile for an area level indicator of disadvantage) preschool children are almost $50 \%$ more likely to be overweight or obese compared to the most advantaged children. ${ }^{1}$ Further, the socioeconomic differentials already present at 4-5 years of age had more than doubled by age $10-11$ years. ${ }^{5}$ The reasons children from socioeconomically disadvantaged families have higher rates of obesity are complex and multifactorial. Evidence suggests that predictors of child obesity in early life, such as higher rates of formula feeding, early introduction of solids, unhealthy infant feeding practices and poorer diet are more prevalent in these families. For instance, a recent longitudinal study among socioeconomically disadvantaged families in the USA found that unhealthy infant feeding practices, including early introduction of solids $(<4$ months of age), feeding infants predominately formula for the first 6 months and putting infants to bed with a bottle, were the primary mechanism mediating the relationship between socioeconomic disadvantage and early childhood obesity. ${ }^{4}$ This suggests that children from socioeconomically disadvantaged backgrounds have a higher exposure to obesity promoting environments and are in greater need of support to establish healthy behaviours early in life. For example, a feeding and activity-based intervention targeting infant of first time mothers in Victoria, Australia (InFANT) showed that parental education level was an important mediator of the intervention effects. ${ }^{6}$ This suggests that new avenues are needed to address the specific needs and challenges faced by disadvantaged families to ensure intervention approaches are effective.

Despite the importance of early preventive efforts directed at socioeconomically disadvantaged families, the most effective approaches for reaching these families are unknown. Primary Health Care services (PHC), including maternal and child health services and general practice are frequently visited by parents across socio-demographic groups. PHC practitioners are highly engaged with parents, offering advice on feeding and settling strategies immediately after birth, but may not be specifically targeting behaviours that promote excess weight gain. ${ }^{7}$ On average, parents in Australia make 11 visits to general practitioners and 14 visits to Maternal and Child Health $(\mathrm{MCH})$ nurses in the first year of their child's life. ${ }^{8}$ Because the majority of these visits are unrelated to illness there is a key opportunity for intervention at a time when parents are potentially more or most receptive to health promoting advice. Practitioners report a high level of interest in obesity prevention among parents, but report a wide range of barriers to delivering obesity prevention support, including system level barriers (lack of time, remuneration, support staff, appropriate resources and programs for referral), attitudinal barriers (concern about parental reactions, discomfort raising the issue), and a lack of knowledge, skills and training in the area. ${ }^{9-12}$

One emerging and promising avenue for delivering obesity prevention initiatives to low SES families involves intervening via electronic media such as the Internet or smart phones (m- or e-health interventions). Evidence suggests that mobile phones are uniquely positioned to bridge gaps in health disparities and to enable access across demographics ${ }^{13}$ because they provide an opportunity to provide high quality information and practical support economically. Almost every adult Australian (up to 60 years of age), including those with low incomes or in low-status occupations, owns at least one mobile telephone $^{14} ; 68 \%$ own a smartphone (ie, mobile telephones with advanced capabilities such as Internet and apps), and with smartphone costs continually decreasing, it is estimated that in 3 years almost all Australian adults will own a smartphone. ${ }^{15}$ In Australia, the majority $(61 \%)$ of smartphone owners are 40 years of age or younger. ${ }^{16}$ Of particular promise, and contrary to popular belief, in countries with high penetration of wireless technology including Australia, income is not a predictor of mobile telephone or smartphone ownership. ${ }^{17}$ There is also evidence to suggest that parents use the Internet and smart phone apps as an important source of information on infant feeding. ${ }^{18}$ However a recent study found the quality of websites and apps on infant feeding available in Australia to be generally poor, ${ }^{19}$ suggesting that parents may not be receiving accurate information from these sources. Early research on the effectiveness of $\mathrm{m}$ interventions in changing health behaviour is promising ${ }^{20}{ }^{21}$ however there is a paucity of research in the area of obesity prevention in infants.

This paper describes the development and protocol for the Growing healthy study, a non-randomised quasi experimental study initiated in PHC examining the feasibility of an intervention delivered via a smartphone app (or website) for parents living in socioeconomically disadvantaged areas on infant feeding and parenting behaviours that promote healthy rather than excessive weight gain.

\section{STUDY AIMS}

The aims of this study are to assess:

1. The feasibility of PHC practitioners referring parents to and incorporating an m-health intervention and reinforcing key messages as part of routine baby health checks;

2. The effectiveness of an m-health intervention in terms of its reach, use, acceptability, cost and impact on key infant nutrition and feeding outcomes. 


\section{METHODS AND ANALYSIS}

\section{Intervention development}

The process for developing the intervention has been informed by intervention mapping ${ }^{22}$ which involves five key steps: (1) developing program objectives, (2) selecting theory-based intervention methods and practical strategies, (3) designing and organising a program, (4) specifying adoption and implementation plans, and (5) generating program evaluation plans. The selection of program objectives, theory based intervention methods and practical strategies was based on the findings from three sources: (1) systematic reviews of the literature, (2) qualitative interviews with socioeconomically disadvantaged mothers, and (3) questionnaires and interviews with maternal and child health nurses and nurses in general practice. Key determinants (classified as relating to capability, opportunity or motivation) of each of the target behaviours were linked to behaviour change techniques using the Behaviour Change Wheel (BCW), a framework for designing and evaluating behaviour change interventions. ${ }^{23}$

\section{Systematic reviews of the literature}

Two systematic reviews were conducted. One review examined the effects of parent and child behaviours on overweight and obesity in disadvantaged children, and identified considerable gaps in the evidence base linking socioeconomic disadvantage or Indigenous status to overweight and obesity, especially with regards to formula feeding. Notwithstanding the limited evidence available, the importance of tailoring interventions to specific socio-demographic groups due to the links between, for example, ethnicity or parental education level and obesity-related parenting behaviours and infant weight outcomes was a relevant finding. The second review examined the effectiveness of interventions in promoting healthy weight in children $0-5$ years from socioeconomically disadvantaged and Indigenous families. ${ }^{24}$ The findings of that review suggest that anticipatory guidance approaches in infancy (generally from birth or antenatally) appear to be effective in influencing early obesity related behaviours such as breastfeeding or the timing of introduction of solids. However the results also indicated that interventions may need to commence in the antenatal period or at birth to positively impact on breastfeeding outcomes amongst socioeconomically disadvantaged mothers.

\section{Qualitative interviews with parents}

We also conducted qualitative interviews with socioeconomically disadvantaged mothers to understand the malleable determinants of each of the intervention's target behaviours, as well as their mobile phone use and attitudes to an m-health program, using the COM-B framework $^{23}$ as a guide. Results showed that the target behaviours were affected in different ways by mothers' capability (eg, knowledge about the benefits of the behaviour, skills in performing the behaviour), opportunity (eg, support and advice provided by health professionals or family) and motivation (eg, desire to perform the behaviour, forming plans and goals). These results informed the selection of relevant Behaviour Change Techniques tailored to each of the target behaviours. $^{23}$

\section{Survey and interviews with nurses}

Maternal and Child Health nurses from two local government areas in the outer metropolitan areas of Melbourne in Victoria, and in the Illawarra/Shoalhaven Medicare local district (a semi-rural, coastal community south of Sydney) in NSW were invited to complete an online survey. The survey solicited data to establish nurses' current practices when caring for parents with infants, with an emphasis on feeding, settling and parenting practices. Nurses were also asked about their perceived role in infant and child obesity prevention and a subset of respondents agreed to be interviewed and these participants were asked more in-depth questions about their current practice. Findings from this study suggest that nurses are well placed to address healthy infant feeding practices because they are frequently consulted for advice, develop strong relationships with parents and preventive care fits well with their perceptions of their current role. ${ }^{7}$

\section{Focus groups with parents}

Four focus groups with 6-8 participants were conducted. All of the focus group participants were mothers, and they were asked to provide feedback on the appearance and content of the app as well as their preferences for frequency and content of the push notifications. Push notifications allow the Growing healthy app to notify a user of new messages or events even when the user is not actively using the application. The message would appear in the notification center or on the lock screen on iOS devices and on the status on Android devices.

The participants indicated that they would like to receive information that was specific to them and their infant (eg, milestone based, or reminders about immunisation) and two to three notifications each week was the preferred frequency. Mothers who were not breastfeeding did not want to receive any messages about breastfeeding and participants reported that they would be 'turned off' by breastfeeding messages. Mothers preferred messages that were perceived as positive, affirming and personalised.

\section{Focus groups with practitioners}

Members of the research team attended a Maternal and Child Health nurses' staff development day to demonstrate the Growing healthy app and invite feedback. The nurses made recommendations on the appearance of the app and the way the content was arranged. They were particularly asked to comment on whether the app content was consistent with their current practice and if they would be comfortable recommending the app to 
parents. In general, the nurses recommended that the photographs contained in the app reflect current recommendations around infant feeding (pictures of breastfeeding rather than formula feeding) and sleep (positioned on back rather than front or side). The nurses acknowledged that the content was consistent with guidelines and agreed to participate in the study and refer parents to the app.

\section{STUDY DESIGN}

The study will use a quasi-experimental design with an m-health intervention group and a concurrent nonrandomised comparison group recruited via online forums. The comparison group will complete the same parent surveys (infant age less than 3 months, 6 months and 9 months) as the intervention group. It was not possible within the project budget to conduct a randomised controlled trial and the comparison group will provide a useful point of reference in terms of assessing the impact of the program on infant feeding outcomes. We will attempt to recruit a group of parents with similar socio-demographic characteristics to the intervention group. Any differences in baseline responses between intervention and comparison groups will be controlled for in the analysis.

\section{STUDY SETTING-INTERVENTION ARM}

The m-health intervention will be tested among 200 parents from socioeconomically disadvantaged communities in three settings: (1) maternal and child health services in three local government areas in Melbourne, Victoria, (2) Outpatient antenatal services at a large Melbourne hospital, (3) General practices in the Illawarra/Shoalhaven Medicare local in NSW.

These settings have been chosen to test the feasibility of integrating the intervention program across different primary health care settings. Choice of study sites was influenced by relative level of socioeconomic disadvantage in the surrounding communities (based on an area wide indicator, the socioeconomic index for $\operatorname{areas}^{25}$ ) as well as birth rate, involvement in previous studies and proximity to the study researchers.

Services and practices will initially be approached by the lead researchers to gauge their interest in participating in the study. Participating staff from these settings will be engaged for the purposes of recruiting parents to the intervention arm and to reinforce key intervention messages as part of routine consultations. Staff will attend a briefing session which will cover the purposes of the study, the content of the intervention program and the process for recruitment and enrolment of parents.

\section{RECRUITMENT AND ENROLIMENT-INTERVENTION ARM}

Eligibility criteria for participation in the intervention arm include:
- Pregnant (30+ weeks gestation) or parent/main carer of an infant aged under 3 months

- Own any type of mobile phone

- Can speak and read English

- Are aged 18 years or older

- Live in Australia.

We plan to use a number of recruitment strategies to encourage participants into the intervention. Multiple methods have been chosen to gauge the effectiveness in reaching the target population and to inform recruitment methods for subsequent trials. These include:

- In the participating areas, the maternal and child health nurses, antenatal staff or practice nurses will give potential participants a program brochure

- Posters will be displayed in waiting rooms in participating clinics/centres/practices

- Parents attending maternal and child health services will be invited to complete an expression of interest form. On these forms parents will provide contact details and give permission for the research team to email information about the study to them

- A research assistant will inform parents about the study at first time parent groups in the selected low socioeconomic suburbs

- Letters will be sent to parents with infants less than 3 months of age or to women in their final trimester of pregnancy (General practices in NSW only)

- Advertisements on parent-centric websites.

Participants will enrol online via the study website http://www.growinghealthy.org.au. This will involve completing a screening form, followed by providing consent and completion of the baseline survey. Participants will then receive a code to download the app (at no cost) from the App Store (iPhone users) or Google Play (Android users) or a login for the website (for those without a smart phone capable of supporting the app). Participants will receive a $\$ 20$ gift voucher after surveys are completed at baseline, 6 and 9 months.

Expectant mothers interested in participating in the study will register their interest on the study website. These mothers will receive a text message/email inviting them to enrol in the study on the study website (as detailed above) 2 weeks after their infant's due date.

\section{REMINDERS}

Reminder emails will be sent to the following groups:

- Those who enroll in the intervention program but do not activate the app (via entering the code)—this will be sent 1 week after enrolment

- Those who express interest in the intervention program but do not enroll-this will be sent 1 week after the initial email.

\section{COMPARISON GROUP}

We will recruit a comparison group online using a range of forums including parenting blogs, parenting websites and Facebook. Online posts will include a link to a 
survey. This will include an online screening form, followed by a consent form and then the baseline survey. Participants who complete the baseline survey will be considered enrolled in the study and followed up when their infants are 6 and 9 months old to complete additional surveys. Participants will receive a $\$ 40$ gift voucher following the completion of the final survey. The comparison group will not receive any intervention (usual care). Reminder emails will be sent to comparison group participants when the 6-month and 9-month survey are due for completion.

\section{SAMPLE SIZE}

The study will recruit 200 parent/child dyads to the intervention arm. This number is based on an anticipated recruitment rate of $25 \%$ of the births in the local government areas, allowing 6 months for recruitment. A similar number will be recruited to the comparison arm via online forums. As this is a feasibility study, the sample size is not based on a statistical power calculation; rather the purpose of the study is to test feasibility in three different primary care settings, and so sample size is tailored to logistical limitations inherent in the different trial settings. Nevertheless, the data gathered in this study will provide evidence to guide sample size calculations for subsequent randomised trials.

\section{THE GROWING HEALTHY INTERVENTION}

The Growing Healthy program is a new app, website and online forum providing parents with a 'one-stop shop' for evidence based advice and tips, consistent with national guidelines on infant feeding in the first 9 months of life. The aims of the program are to:

- Promote breastfeeding

- If breastfeeding is not possible, promote best practice formula feeding

- Delay the introduction of solids to around 6 months of age but not before 4 months

- Promote healthy first foods

- Promote healthy infant feeding practices (including feeding to appetite, repeated neutral exposure to healthy food and avoiding using food as a reward)

- Optimise infant dietary exposure to fruits and vegetables.

A summary of the aims and strategies of the program can be found in table 1 .

Participating parents will receive three push notifications in the phone app for each week of the intervention on infant feeding topics relevant to the age of their infant. Messages will be tailored to their feeding mode (breast, formula or mixed) with links provided to more information on the app/website (http://www. growinghealthy.org.au). Parents will also have the opportunity to connect with other parents on the Growing healthy Facebook group. This will be a 'secret group', meaning that it is only available to those participants who consent to joining the Facebook group. Three messages a week will also be posted to Facebook to reinforce program content and to encourage interaction and engagement with the program.

\section{DATA COLLECTION}

\section{Parent surveys}

Both intervention and comparison participants will be asked to complete surveys at baseline (upon enrolment in the study when their infant is less than 6 months of age), and when infants are aged 6 and 9 months. Data will be collected on sociodemographic characteristics, infant feeding practices and perceived usefulness of intervention components (in Growing healthy participants only). A summary of the domains at each time point is included in table 2.

\section{Breastfeeding}

At baseline, questions regarding experience of initiation, self-efficacy and supplementation will be asked of participants who are currently breastfeeding and who initiated but have ceased breastfeeding. Participants who are currently breastfeeding (at baseline and 3 months) will be asked questions regarding their experience and practices and sources of support. Those parents who have stopped breastfeeding will be asked about age of weaning and reasons for weaning.

\section{Formula feeding}

Participants will be asked to complete seven items from the Baby Milk Study ${ }^{26}$ on the brand of formula chosen, response to satiety cues and the methods of preparation. They will also be asked to complete questions derived from the questionnaire developed by Baughcum regarding formula feeding practices. ${ }^{27}$

\section{Solids and dietary exposure to fruit, vegetables, non-core snacks and drinks}

At the baseline and 6-month surveys parents will be asked about the time at which they introduced solid foods and fluids other than breast milk or formula. At 6 months, participants will be asked one item about the reasons for introduction of solid foods and another item about type of foods offered in the first month of eating. At 9 months parents will be asked about sources and usefulness of advice on solids (1 item). The 9-month survey will include a purpose designed tool to measure dietary exposure, taste preference and intention to offer specific foods in the next 6 months. Food items for which these variables will be measured are fruit (10 items), vegetables (10 items), core and non-core drinks (5 items), non-core snacks ( 7 items). For each food item parents will be asked about frequency of offering these foods (never, less than once a month, 1-3 times a month, once a week, 2-4 times a week, 5-6 times a week, once a day or more), 'does the child usually like the food' (yes, no, hasn't tried this food) and 'will you offer this food in the next 6 months (yes, no, unsure). The aim of the 


\section{Program objectives (target behaviours)}

1. Promote breastfeeding

A. Mother exclusively breastfeeds until the introduction of solid foods

B. Mother continues breastfeeding alongside introduction of solid foods and other liquids - for the duration of the intervention (9 months)

C. Mother provides breast/formula combination over formula exclusively before and after the introduction of solid foods ('mixed feeding')

2. Best practice formula feeding

A. Mother chooses the most appropriate formula for the infant

Formula if often incorrectly prepared (capability)

B. Mother prepares formula correctly Cereal is added to formula to promote (follows instructions on tin for loosely sleep (motivation) packed, level scoop, correct number of

\section{Key determinants}

(theoretical domain)

Able to breastfeed without pain/ problems

Getting appropriate help when needed BF frequently (enough to maintain milk supply)

Having enough milk

Believing have enough milk

(confidence)

Not introduce formula early/supplement with formula

Commit to breastfeeding exclusively

(plan/intend)

Be able to breastfeed in public as

needed

Avoid introducing dummy early/correct

dummy use

Continuing breastfeeding after returning

to work

Manage realistic expectations

Supportive partner/family/peers

\section{Sample strategies}

(intervention function)

Push notification/text message

Training (latch, dummy use)

Education (breastmilk as normal food for

babies, milk supply cycle, checking if

baby is getting enough)

Support (providing contact numbers for

help, information on realistic

expectations)

Motivate (via messages to set

breastfeeding goals, to continue

breastfeeding, to seek help and support)
Do you want to breastfeed (y) but find it painful? Ask for help! Most problems can be worked through with the right help. Find help here. (Links to help, baby age 2 weeks)

If you are thinking of trying a dummyTry waiting 1 more week till you set up your milk supply, and learn the best way to use one here. (Links to

information on dummies, baby age 3 weeks)

Did you know that the more you feed the more milk you make? Read more about milk supply here. (Links to information on milk supply, baby age 5 weeks)

How is breastfeeding going? It gets easier as your baby gets older! Focus on your successes knowing you're doing the best thing for your baby (links to information on why to breastfeed, baby age 6 weeks)
Model correct formula preparation using a video (modelling)

Educate that adding cereal to the bottle will not help baby sleep (educate)

Demonstrate settling strategies (training) and provide information on how to
Able to make formula in your sleep? Don't forget-always add the water THEN the powder when making up a bottle. More on making formula here (link to step by step instructions and video-baby age 5 weeks) 


\section{Program objectives (target behaviours)}

scoops, uses correct scoop, add water first)

C. Mother does not add anything else to the bottle (eg, cereals, honey)

D. Mother uses appropriate feeding practices (ie, cradles baby throughout feed, no bottle cropping, doesn't put baby to bed with bottle)

E. Initiates feeding according to baby's hunger level. Does not force baby to start feeding

F. Stops feeding according to baby's hunger level. Doesn't force continuation (stops feeding when infant loses interest, not when formula is finished or a certain volume is consumed)

G. Mother does not use milk to sooth infant

H. Mother does not give infant formula to promote sleep (by reducing hunger)

3. Delay introduction

A. Mother begins regular (regular=more than twice a week for several continuous weeks) feeding of solid foods (ie, anything other than breast milk or formula) around 6 months of
Key determinants

(theoretical domain)

to infant crying (motivation/capability)

Baby is put to bed with a bottle to

promote sleep (motivation/capability)

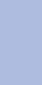

\section{Sample strategies}

(intervention function)

promote better sleep for baby (educate)

without use of milk for settling

Demonstrate hunger, fullness and sleep cues (education/training)

Provide information on baby's ability to

self-regulate appetite (education)

Provide information on dangers of putting baby to bed with bottle (educate) and strategies to promote better sleep (training)

Provide information on dangers of bottle propping (education) and benefits of holding baby while feeding

(incentivisation)
Belief that of solids to around 6 months but not before 4 months introduction of solids will help baby sleep through the night (motivation) Normative influence-everyone else seems to be starting solids at 4 months (motivation)
Provide information and demonstrate skills to promote sleep and settling without non-nutritive feeding (education/ training)

Provide information about consequences of early introduction of solids and benefits of delaying to 6 months (persuasion)

Provide information about signs of readiness to introduce solids (education) Provide support in managing other peoples expectation to start solids before 6 months (enablement)

\section{Push notification/text message}

When (y) is crying do you find yourself reaching for the bottle sometimes?

Read here for other ways to manage the crying (baby age 4 weeks) Does (y) drink different amounts of formula on different days? This is normal! Read more on how much to feed here (baby age 13 weeks)

Tempted to put (y) to bed with a bottle $(x)$ ? It's best not to if possible. Find out why here (baby age 27 weeks) Tempted to prop (y) up with a bottle? This can be unsafe and you'd miss the chance for a cuddle! Read more on bottle feeding here (baby age 16 weeks)
Been told different advice on when to start solids? You're not alone. Watch the advice a dietitian gives a mum experiencing the same here (16 weeks) Did you know starting solids early can affect your baby's health?

Until 6 months, milk is all $(\mathrm{y})$ needs See more on starting solids (baby age 13 weeks)

Did you know that introducing solids before 6 months probably won't help (y) sleep at night?

See some tips on sleep here (baby age 14 weeks)

Wondering if it's finally time to introduce solids? There are a few things that $(\mathrm{y})$ might start doing when it's time-see here (baby age 20 weeks) 
Table 1 Continued

\section{Program objectives (target behaviours)}

Key determinants

(theoretical domain)

4. Healthy first foods

A. Mother introduces healthy iron rich Lack of cooking skills and reliance foods as first foods (eg, infant rice cereal, vegetables) (first foods defined as those foods that infants are given in the first month of eating solids)

B. Mother doesn't introduce unhealthy high caloric, energy dense foods and drinks (eg, processed foods) as first foods

C. Appropriate transition to family foods

\section{on commercial baby food}

- Lack of knowledge about high iron foods and how to prepare them
5. Promotes infant feeding practices for healthy growth

Mother exposes child to healthy new foods

(exposure)

A. Mother repeatedly exposes child to healthy foods, even if such foods are initially rejected by the child (repeated exposure).

B. Mother feeds to appetite, that is, does not pressure child to eat more than $s /$ he wants (pressure to eat)

Lack of knowledge about food preference development and ability to self-regulate (capability)

- Concern that baby is not getting (motivation)

\section{Sample strategies}

(intervention function)

Push notification/text message

Demonstration cooking videos and recipes on how to prepare meals for baby based on family meals (education/ training)

Provide information on high iron foods and how they can be prepared (education /training)

Provide information about and demonstrate with cooking videos how to change texture of foods for baby
Guess what $(x)$ ? It's time to start $(y)$ on solids! First foods should be high in iron, like meat, fish or lentils-read more (baby age 22 weeks) How are the solids going? Remember to keep on trying different healthy foods and to avoid those high in fat, sugar or salt. Some options here (baby age 25 weeks)

Isn't (y) growing quickly?

Make sure you change (y)'s food texture to help develop chewing and swallowing muscles. Read more here (baby age 29 weeks)

Are you stuck on what to feed (y) at each meal? Tap here for a recipe idea, you can use for the whole family! (baby age 33 weeks)

Normalise food rejection and provide strategies to manage (education/ training) addressing 'fussy eating' (capability)

- Parents not aware of infants innate enough milk/food for growth or sleep
- Provide information about baby sleep patterns and non-feeding approaches to promoting sleep education/training)
Does (y) spit out new foods? This is normal! It can take 10-15 tries for babies to eat new foods-keep going! More on food rejection (baby age 26 weeks)

Wondering how much you should feed (y)? Tap here to learn about 'Parents Provide, Babies Decide' (baby age 33 weeks) 
Table 2 Questionnaire domains at time points

\begin{tabular}{|c|c|c|c|}
\hline Measures & $\begin{array}{l}\text { T1 (<3 months } \\
\text { of age) }\end{array}$ & $\begin{array}{l}\text { T2 (6 months } \\
\text { of age) }\end{array}$ & $\begin{array}{l}\text { T3 (9 months } \\
\text { of age) }\end{array}$ \\
\hline \multicolumn{4}{|l|}{ Breastfeeding } \\
\hline Exclusive breastfeeding & $\mathrm{X}$ & $\mathrm{X}$ & \\
\hline Breastfeeding duration & $\mathrm{X}$ & $\mathrm{X}$ & $\mathrm{X}$ \\
\hline Reasons for replacing/supplementing breast milk & $\mathrm{X}$ & $X$ & \\
\hline Breastfeeding self-efficacy & $\mathrm{X}$ & & \\
\hline Age of dummy introduction & $\mathrm{X}$ & & \\
\hline BF advice received & & $\mathrm{X}$ & \\
\hline \multicolumn{4}{|l|}{ Formula Feeding } \\
\hline Formula type and amount given & $\mathrm{X}$ & $\mathrm{X}$ & \\
\hline Formula preparation & $x$ & $X$ & \\
\hline Formula feeding practices & $x$ & $\mathrm{X}$ & \\
\hline Formula feeding advice received & & $X$ & \\
\hline \multicolumn{4}{|l|}{ Solids and diet quality } \\
\hline Timing of introduction of solids & & $\mathrm{X}$ & \\
\hline First foods & & $\mathrm{X}$ & \\
\hline Additional fluids (not water or breast/formula milk) & $\mathrm{X}$ & $X$ & \\
\hline Reason for solids introduction & & $X$ & \\
\hline Advice on solids received & & & $\mathrm{X}$ \\
\hline Diet quality (FFQ) & & & $\mathrm{X}$ \\
\hline Child food preferences (FFQ) & & & $\mathrm{X}$ \\
\hline Parental Intentions to re-offer disliked food & & & $X$ \\
\hline \multicolumn{4}{|l|}{ Parental feeding behaviours } \\
\hline Infant satiety scale & $\mathrm{X}$ & $\mathrm{X}$ & $\mathrm{X}$ \\
\hline Baughcum Infant Feeding Questionnaire & $\mathrm{X}$ & $\mathrm{X}$ & $\mathrm{X}$ \\
\hline \multicolumn{4}{|l|}{ Anthropometrics } \\
\hline Height & $\mathrm{X}$ & $\mathrm{X}$ & $\mathrm{X}$ \\
\hline Weight & $x$ & $\mathrm{X}$ & $X$ \\
\hline Head circumference & $x$ & $x$ & $x$ \\
\hline
\end{tabular}

tool was to assess dietary exposure, baby's food preferences and parental intentions to continue offering disliked fruit and vegetables (repeated exposure), a key aim of the intervention.

\section{Parental feeding behaviours and beliefs and infant satiety}

To assess parental feeding behaviours and beliefs about infants that may be associated with overweight, participants will be asked at all time points to complete the Infant Feeding Questionnaire. ${ }^{27}$ This questionnaire contains 19 items related to parental feeding (eg, using food to calm a fussy infant) and beliefs (eg, concern about infant's hunger). Participants will also be asked about their concern about their infant's current weight. The infant's ability to respond to internal satiety cues will be measured with three items from the Baby Eating Behaviour Questionnaire subscale Satiety Responsiveness. ${ }^{28}$

\section{Anthropometrics}

Parents will be asked to record the weight and length of their infant at all three time points. At baseline they will be asked to record their infant's birth weight and length, as well as the most recent measurement from their infant health record (also called the 'blue book' or the 'green book'). Mothers will be asked their height and pre-pregnancy weight at baseline.

\section{Sociodemographic characteristics}

Participants will be asked to report on standard demographic measures at baseline including country of birth, age, postcode of residence, relationship status, employment status, family income and education level.

\section{Health economics}

The cost benefit of the Growing healthy program will be assessed by asking participants questions at 9 months regarding their usage and costs of health care professionals. The Growing Healthy intervention, through provision of information, may decrease (or increase) the parent's use of health services to gain help with respect to feeding, activity and weight issues. To measure these effects, both the Growing Healthy group and the control group will be asked to report their use of services related to their infant's or their own weight, diet or activity since their baby's birth to capture differences in service usage. Participants will be asked about the use of maternal and child health nurses, parenting centres as well as the family doctor, paediatrician, dietician and other health professionals. Parents will be asked to report the number of visits to services used and any out-of-pocket costs. 


\section{Process evaluation data}

Both the app and the accompanying website incorporate data capture technology to enable analytics to support the operational aspects of the intervention and to provide quantitative evidence of the intervention's success. The analytics is achieved through tight integration between the app, the website and the backend servers including the management of user profiles, feeding methods, and serving of personalised pushnotifications/text messages throughout the entire study period.

This allows for fine granular data to be captured on the server, which can be used to help the research team answer different questions about the engagement of the participants, management operational issues such as follow-up on sign-up, surveys and program support. Currently, the raw data capture includes:

- Profile information of participants, including baby's age, mum's contact details and unique activation code that is used to identify a user

- App activities, including when a participant viewed a particular content such as a video or a page in the app, when a push-notification was tapped, and when a survey was started. For the user group who are receiving text and email messages, the same type of data is replicated whenever possible but obviously, the data will not be as sophisticated as that of the app user because of the nature of text (SMS) and email technology

- Log of activations (for app users) and logins (for mums who are on text/email messaging)

- A database of mums who registered interest to join the program after the birth of their child.

The raw data $\log$ is useful when combined with analytical tools to help enhance the operational aspects of the intervention, and to manage (and also measure) the engagement throughout the entire period of study. For example, the research team is able to see which pages are the most popular or least favourite among the participants. The same insight can be obtained for videos and personalised push notification messages, containing the crucial intervention messages. More powerful analysis can be obtained when the fine grained log is processed through a number of other variables, such as how the popularity of videos change when broken down by the feeding methods or over time.

These insights will provide the research team an ongoing and on-demand access to information about how the participants are engaging in the program throughout the study period. As participants progress through the study, the analytics will provide insights into how mums are engaging with the app (or the website) over the study period. This will help fully measure the engagement extent of the program.

\section{Parent interviews (Growing healthy only)}

One-on-one semi-structured telephone interviews will be conducted with a purposeful sample of parents participating in the intervention program (high and low program users, $n=$ approx 30) following the 9-month survey. The aim of the interviews is to explore parents' experience of using the program, acceptability, and factors related to engagement. An interview guide will be developed around an engagement framework to explore the features of the program affecting participants' engagement levels (eg, appropriateness of content, usability of app) and outcome behaviours.

\section{Practitioner surveys and interviews}

Practitioners participating in the Growing healthy program will be ask to complete a brief 5-10-min survey, 6-12 months after program commencement. The survey will aim to gather feedback on the recruitment process and the program itself. Qualitative interviews will be conducted with a purposeful sample of participants (across practitioner types) to further explore nurses views on the feasibility of the parent recruitment approach and reinforcement of key messages as part of routine consultations. The interviews will also explore nurses views on the intervention including credibility, perceived need, usefulness, and suggestions for improvement.

\section{DATA ANALYSIS}

\section{Quantitative data}

Baseline data will be analysed descriptively by group. Appropriate statistical methods will be used to compare the following infant feeding outcomes between intervention and comparison groups:

- Duration of breastfeeding

- Formula feeding practices

- Age of introduction of solids

- Quality of first foods

- Parental feeding behaviours

Exposure to fruits, vegetables and non-core foods. Survival analysis will be used for analysis of breastfeeding duration and age of introduction of solids. The other outcomes which are categorical in nature will be analysed using generalised linear models, particularly logistic regression (binary, multinomial and ordinal).

Secondary analysis will adjust for potential confounders including parent's age, education and income. Descriptive statistics will be used to analyse process data on program feedback such as the usefulness of program components.

Analyses will be performed using appropriate statistical software such as SAS (SAS Institute, Cary, North Carolina, USA) and/or SPSS (IBM SPSS Statistics, Armonk, New York, USA).

Qualitative data will be analysed using a thematic approach and triangulated with quantitative data (where possible) to answer the research question.

An economic evaluation of the Growing healthy program will be undertaken using the outcomes (consequences) data described above and costs. The first task will involve identifying, measuring and valuing the 
relevant costs and consequences of the Growing healthy participants (intervention) and the control group. The total costs include the cost of resources used associated with the app (and website) such as server hosting and moderator, as well as cost of health services used. Quantities of resource use will be measured and unit costs (prices) will be assigned, using current pay rates, commercial rates (prices) and individuals' reported out-of-pocket costs. The specific consequences related to the infant feeding outcomes to be explored in the evaluation are: (a) median duration of breastfeeding, (b) proportions who correctly prepare formula; proportions who add anything else to formula, (c) median duration of introduction of solids, (d) proportion who use iron rich foods as first foods, (e) proportion who feed to appetite, (f) proportion who repeat exposure to healthy foods, (g) proportion of children who receive foods from four or more food groups. All outcomes (consequences) will be measured in natural units.

The second task will involve comparing changes in the total costs and total outcomes for these groups. This will comprise an incremental analysis of the costs and consequences of the Growing healthy intervention; comparing the costs associated with the Growing healthy program over the control group with the additional outcomes generated by the intervention in terms of the outcomes already described. The results of the costs and outcomes will be presented separately (ie, in a cost-consequence analysis) and in several cost-effectiveness ratios (such as a cost per unit change in age of introduction of solids).

\section{Qualitative data}

All qualitative interviews will be audio recorded with participant's permission and transcribed verbatim. A thematic analysis informed by the methods of Braun and Clarke will be undertaken to identify common and divergent themes. Nvivo 10 will be used for data coding, sorting and retrieval.

\section{DISCUSSION}

The Growing healthy study, to our knowledge, is the first m-health intervention targeting infant feeding and parenting behaviours. The intervention content was developed by experts in the field to complement routine management by PHC providers. It is evidence based and available on a smart phone supported app or website and includes three age appropriate messages each week. This study will address an important gap by developing a novel m-health intervention addressing nutrition and obesity risk for disadvantaged families. The study will examine how such an intervention can be delivered within the PHC setting, including the most effective approach to reaching disadvantaged families, as well as the feasibility, uptake, effectiveness and sustainability of the intervention from the perspective of both families and practitioners.

Given the lack of evidence regarding effective interventions for this target group, particularly within the
PHC setting, this work (akin to a Phase 2 trial) is especially crucial to public health trials as it informs robust, achievable and ethically designed full efficacy trials.

\section{STUDY LIMITATION}

The study, at the outset, has a number of limitations. It is using a non-randomised concurrent comparison group. The socio-demographic profile of the comparison group may be different to the intervention group, particularly given that the comparison group will be recruited solely through online sources. This may limit comparison between groups on key outcomes, however some of these differences can be controlled for in the analysis. A more robust RCT or cluster RCT design was not possible within the study budget and timeframe and thus the aim of the study was to collect data primarily on feasibility to inform a full scale RCT in the future. The length of follow up for this study is limited to 9 months of age again due to study budget and timeframe. Ideally longer term follow up would be preferable to allow for the effect of the intervention to be assessed in later infancy and toddlerhood. A further limitation is a lack of objectively measured anthropometrics or infant feeding practices.

\section{PLANS FOR DISSEMINATION OF FINDINGS}

The Growing healthy results will be submitted to peerreview journals for consideration. The research team will also submit abstracts to local and international conferences and will provide feedback via workshops for clinicians and online newsletters (for parents).

Twitter Follow Elizabeth Denney-Wilson at @denneywilson

Acknowledgements The authors wish to thank the parents and nurses who participated in questionnaires, focus groups and interviews. We also thank Professor Cathrine Fowler who reviewed the app content for accuracy and consistency with guidelines and Ms Louisa Wilson who proof read the manuscript.

Contributors ED-W, RL, CGR and KC conceived and designed the study. $\mathrm{ED}-\mathrm{W}, \mathrm{RL}, \mathrm{KC}, \mathrm{JL}, \mathrm{DC}, \mathrm{KB}$ wrote the project grant and were awarded funding for the research. ED-W and RL wrote the first draft of the manuscript and CGR, KL, ST, RE, LA, SL, RT, JL, DC, KB, DA and EKL provided specific content and edited the manuscript.

Funding This work was supported by a grant from the Australian Primary Health Care Research Institute (APHCRI) which is supported by a grant from the Australian Government Department of Health and Ageing. APHCRI was not involved in the study design or implementation or in the preparation of this manuscript. KB is supported by a National Health and Medical Research Council Principal Research Fellowship, IS 1042442. The contents of this manuscript are the responsibility of the authors and do not reflect the views of the NHMRC.

Competing interests None declared.

Ethics approval The University of Technology Sydney human research ethics committee and the Deakin University human research ethics committee.

Provenance and peer review Not commissioned; externally peer reviewed.

Open Access This is an Open Access article distributed in accordance with the Creative Commons Attribution Non Commercial (CC BY-NC 4.0) license, 
which permits others to distribute, remix, adapt, build upon this work noncommercially, and license their derivative works on different terms, provided the original work is properly cited and the use is non-commercial. See: http:// creativecommons.org/licenses/by-nc/4.0/

\section{REFERENCES}

1. Wake $\mathrm{M}$, Hardy $\mathrm{P}$, Canterford L, et al. Overweight, obesity and girth of Australian preschoolers: Prevalence and socio-economic correlates. Int J Obes (Lond) 2007;31:1044-51.

2. Askie LM, Baur LA, Campbell K, et al. The Early Prevention of Obesity in CHildren (EPOCH) Collaboration-an individual patient data prospective meta-analysis. BMC Public Health 2010;10:728.

3. Ong KK. Size at birth, postnatal growth and risk of obesity. Horm Res 2006;65(Suppl. 3):65-9.

4. Gibbs BG, Forste R. Socioeconomic status, infant feeding practices and early childhood obesity. Pediatr Obes 2014;9:135-46.

5. Jansen PW, Mensah FK, Nicholson JM, et al. Family and neighbourhood socioeconomic inequalities in childhood trajectories of $\mathrm{BMI}$ and overweight: longitudinal study of australian children. PLOS ONE 2013;8:e69676.

6. Cameron AJ, Ball K, Hesketh KD, et al. Variation in outcomes of the Melbourne Infant, Feeding, Activity and Nutrition Trial (InFANT) Program according to maternal education and age. Prev Med 2014;58:58-63.

7. Laws R, Campbell KJ, Van Der Pligt $\mathrm{P}$, et al. Obesity prevention in early life: an opportunity to better support the role of Maternal and Child Health Nurses in Australia. BMC Nurs 2015;14:26.

8. Goldfeld SR, Wright M, Oberklaid F. Parents, infants and health care: utilization of health services in the first 12 months of life. $J$ Paediatr Child Health 2003;39:249-53.

9. Hearn L, Miller M, Cross D. Engaging primary health care providers in the promotion of healthy weight among young children: barriers and enablers for policy and management. Aust $J$ Prim Health 2007;13:66-79.

10. McMeniman E, Moore R, Yelland M, et al. Childhood obesity: how do Australian general practitioners feel about managing this growing health problem? Aust J Prim Health 2011;17:60-5.

11. Vine M, Hargreaves MB, Briefel RR, et al. Expanding the role of primary care in the prevention and treatment of childhood obesity: a review of clinic-and community-based recommendations and interventions. J Obesity 2013;2013:172035.

12. Robinson A, Denney-Wilson E, Laws R, et al. Child obesity prevention in primary health care: investigating practice nurse roles, attitudes and current practices. J Paediatr Child Health 2013;49: E294-9.
13. Fjeldsoe BS, Marshall AL, Miller YD. Behavior change interventions delivered by mobile telephone short-message service. Am J Prev Med 2009;36:165-73.

14. Australiasian Mobile Telecommunications association. The impact of mobile phone on work-life balance. 2007.

15. Sullivan F. Australian Mobile Devise Usage Trends 2012. In. Edited by Sullivan F. 2012

16. Telstra. Fact Sheet: Telstra Smartphone Index 2011 Highlights. In. Edited by report. Om; 2011.

17. Household Use of Information Technology, Australia, 2012-13.

18. Buultjens M, Robinson P, Milgrom J. Online resources for new mothers: opportunities and challenges for perinatal health professionals. J Perinat Educ 2012;21:99-111.

19. Taki S, Campbell KJ, Russell CG, et al. Infant feeding websites and apps: a systematic assessment of quality and content. Interact $J$ Med Res 2015;4:e18.

20. Free C, Phillips G, Galli L, et al. The effectiveness of mobile-health technology-based health behaviour change or disease management interventions for health care consumers: a systematic review. PLOS Med 2013;10:e1001362.

21. Militello LK, Kelly SA, Melnyk BM. Systematic review of text-messaging interventions to promote healthy behaviors in pediatric and adolescent populations: implications for clinical practice and research. Worldviews Evid Based Nurs 2012;9: 66-77.

22. Bartholomew LK, Parcel GS, Kok G. Intervention mapping: a process for developing theory- and evidence-based health education programs. Health Educ Behav 1998;25:545-63.

23. Michie S, van Stralen MM, West R. The behaviour change wheel: a new method for characterising and designing behaviour change interventions. Implement Sci 2011;6:42.

24. Laws R, Campbell KJ, Van Der Pligt $\mathrm{P}$, et al. The impact of interventions to prevent obesity or improve obesity related behaviours in children ( $0-5$ years) from socioeconomically disadvantaged and/or indigenous families: a systematic review. BMC Public Health 2014;14:779.

25. Census of Population and Housing: Socio-Economic Indexes for Areas (SEIFA), Australia. 2011. [http://www.abs.gov.au/ausstats/ abs@.nst/mf/2033.0.55.001]

26. Lakshman R, Landsbaugh J, Schiff A, et al. Development of a questionnaire to assess maternal attitudes towards infant growth and milk feeding practices. Int J Behav Nutr Phys Act 2011;8:35.

27. Baughcum AE, Powers SW, Johnson SB, et al. Maternal feeding practices and beliefs and their relationships to overweight in early childhood. J Dev Behav Pediatr 2001;22:391-408.

28. Llewellyn $\mathrm{CH}$, van Jaarsveld CHM, Johnson L, et al. Development and factor structure of the Baby Eating Behaviour Questionnaire in the Gemini birth cohort. Appetite 2011;57:388-96. 